\title{
Finite Element Analysis of High Frequency Breaker Vibrator
}

\author{
Xiaofan Hou, Guoping Yang \\ College of Automotive Engineering, Shanghai University of Engineering Science, Shanghai, China \\ Email: billyhouxf@163.com
}

How to cite this paper: Hou, X.F. and Yang, G.P. (2018) Finite Element Analysis of High Frequency Breaker Vibrator. Open Access Library Journal, 5: e4485. https://doi.org/10.4236/oalib.1104485

Received: March 9, 2018

Accepted: April 9, 2018

Published: April 12, 2018

Copyright ( $\odot 2018$ by authors and Open Access Library Inc.

This work is licensed under the Creative Commons Attribution International License (CC BY 4.0).

http://creativecommons.org/licenses/by/4.0/

\begin{abstract}
High-frequency breaker vibrator mainly includes three major components of the structure: the vibration box, knife row and bucket teeth. Due to the periodic impact load of the high-frequency breaker, there is a risk of failure if the structure of the exciter is not designed properly, which will seriously affect the working efficiency and service life of the breaker. The static characteristic of the exciter is an important part of evaluating the overall performance of the breaker. It is necessary to do the statics analysis of the exciter to study the stress distribution and the strain state of the breaker. Based on the above reasons, the stress analysis and strain analysis of the finite element modeling of the exciter structure are carried out in this paper. The simplification and constraint handling of the model are discussed.
\end{abstract}

\section{Subject Areas \\ Mechanical Engineering}

\section{Keywords}

High Frequency Breaker, 3D Modeling, Finite Element Analysis

\section{Introduction}

SV-60-type high-frequency hammer using double eccentric meshing transmission, hydraulic motor driven eccentric rotation generated exciting force $\mathrm{F}$, the vibration box can be relative to the rack compressed air spring. The lower end of the vibrating box transmits the exciting force to the rock through the welded knife row and the teeth of the vibrating box. The vibrating box, the knife row and the teeth of the vibrating box are collectively referred to as the exciter. The vibrating box forms a four-bar linkage mechanism by connecting the frame with 
the pull-up bracket and the pull-down bracket, so that the exciter can be moved horizontally along the vertical plane. This four-bar structure ensures the accuracy of the working point of the breaker and makes the bucket teeth effective Acting on the rock surface [1] [2] [3].

\section{Finite Element Introduction}

For finite element analysis of the analysis we usually boil down to the following steps:

1) Structure discretization

2) Select the displacement mode

The finite element method can be divided into displacement method, force method and hybrid method according to unknown nodes. I choose the displacement method. Due to the displacement method to calculate the automation characteristics make it widely used in the finite element method. In general, in the analysis of continuum problems, we usually assume the distribution of displacements in a cell in order to make the displacement of a node represent the displacement, stress and strain of a cell, that is, the displacement is a function of coordinates, which is a simple function For the displacement mode or interpolation function, usually selected as a polynomial [4]. We can derive the relation of the displacement of any point in the cell represented by the displacement of the node according to the displacement mode:

$$
\{u\}=[N]\{\delta\}^{e}
$$

In the formula, $\{u\}$-displacement array at any point within the cell

$[N]$-Shape function matrix whose elements are functions of the position coordinates

$\{\delta\}^{e}$-The unit's nodal displacement array

3) Analyze the mechanical properties of elements

Select the displacement mode, according to set the number of unit nodes, material properties, geometry and other implications, including the following three parts of the mechanical analysis of the unit:

1) According to the geometric equation, using the displacement expression to derive the node displacement represents the unit strain relation:

$$
\{\xi\}=[B]\{\delta\}^{e}
$$

In the formula, $\{\xi\}$-at any point of the unit within the strain array $[B]$ - Unit strain matrix

2) According to the constitutive equation, the displacement of the node derived from the strain expression (2-2) shows the relation of unit stress:

$$
\{\delta\}=[D]\{\xi\}=[D][B]\{\delta\}^{e}
$$

In the formula, $\{\delta\}$ - any point in the cell stress array

$[D]$ - The elastic matrix related to the material of the unit

3) According to the variational principle, the equilibrium equation of the cell 
is established, that is, the relationship between the node force acting on the element and the node displacement:

$$
\{f\}^{e}=[K]^{e}\{\delta\}^{e}
$$

In the formula, $[K]^{e}$ - element stiffness matrix

4) Set of cell groups to establish structural equilibrium equation

The set of elements consists of a global stiffness matrix that is a collection of element stiffness matrices and a load array that acts as a set of stresses on the equivalent nodes of each element. The direct stiffness method is the most commonly used set stiffness matrix method [5]. The reason for the usual set is based on the requirement that all the adjacent elements have the same displacement at the common node. The equilibrium equation of the whole structure is:

$$
[K]\{\delta\}=\{f\}
$$

where, $[K]$-the overall stiffness matrix

$\{f\}$-for the load array

$\{\delta\}$-Displacement of the array of nodes for the entire object

5) Find unknown node displacement

According to the equilibrium equations (2-5) solve the unknown displacement.

6) Calculate cell stress

According to formula (2-2) and node displacement to calculate the stress of each unit.

\section{Exciter Finite Element Model Establishment}

\subsection{Geometric Model Analysis and Simplification}

Before establishing the finite element model, the exciter geometry model should be analyzed and simplified. In this paper, the geometric model of high-frequency breaker uses CATIA design and assembly of the whole machine. The whole three-dimensional assembly diagram shown in Figure 1.

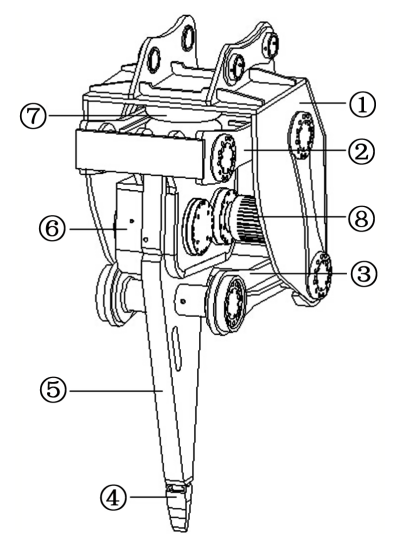

(1)-Rack, (2)—pull up bracket, (3)—pull down bracket, (4)—-tooth teeth, (5)-knife row, (6)-vibration box, (7)-buffer device, (8)-hydraulic motor

Figure 1. SV-60 high frequency breaker structure. 


\subsection{Finite Element Boundary Conditions Established}

1) Load boundary conditions

This article considers two types of exciter load. One is the eccentric block gear rotating at high speed to generate vertical excitation force. One is the excavator to the exciter top load, this load from the excavator weight through the size of the arm and then passed through the air spring [6].

2) Contact boundary conditions

In the finite element model of an assembly, two mutually independent finite element model surfaces touch each other and are tangent to each other. This state is called contact. The geometric contact does not transfer the load during finite element analysis [7]. Depending on the articulation of the pull-up and pull-down brackets with the exciter and the contact characteristics of the CAE CAE platform, we set the articulated contact surfaces as slip connections.

3) Boundary conditions

This paper applies full constraint directly to the bucket end and fixes the top of the exciter with a smooth spring dummy to exert a degree of freedom beyond the axial rotation on the non-hinged ends of the pull-up and pull-down brackets.

Based on the above analysis, the final boundary conditions of exciter components are shown in Figure 2 [8].

\subsection{Finite Element Mesh}

1) Grid definition division

An entity part can have only one definition of an entity grid. The system automatically defines an entity grid for each entity part. The defined entity grids include grid type (cell type, cell order) and size and other information, this grid definition is called grid components [9].

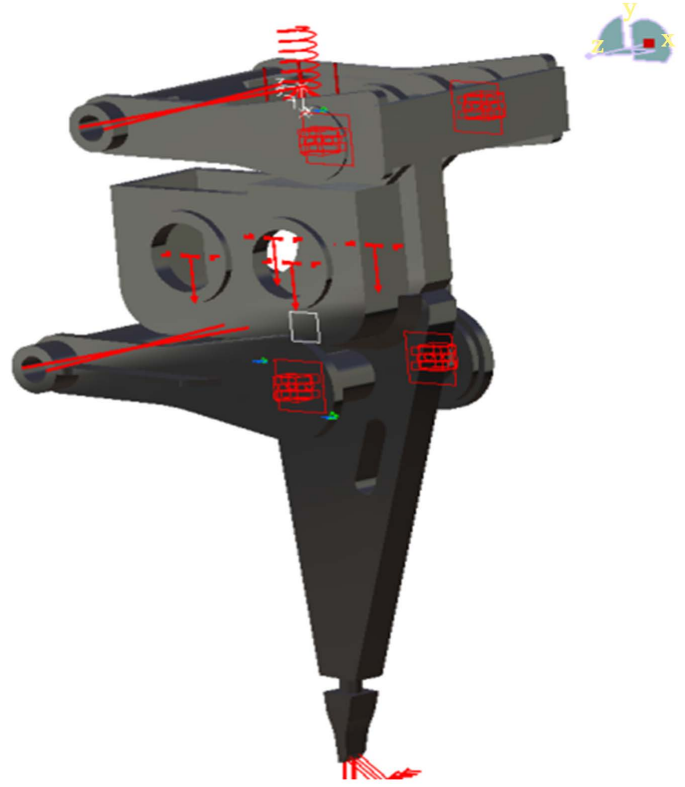

Figure 2. Exciter components boundary conditions. 
2) Automatic grid division and manual division

Manual grid division can be manually adjusted grid parameters and cell structure types, reduce the size of the string and set the unit, using parabolic parabolic edge unit, improve the accuracy of mesh, a total of 278,122 nodes, 166,162 units [10]. Table 1 is the manual meshing quality report. As can be seen, using manual meshing reduced the number of Stretch units to eight, with a percentage of almost zero. Aspect Ratio has 9955 cells, accounting for only 5.88\%. 35.05\% relative to the automatic segmentation results have increased dramatically, the effect is significant [11].

3) Exciter components finite element model

Through the comparison between automatic meshing and manual meshing, and combining the above analysis of the boundary conditions of exciter components and the setting of material properties, the results are shown in Table 2. Select manual division the finite element model of the static exciter finally studied in this paper is shown in Figure 3 [12].

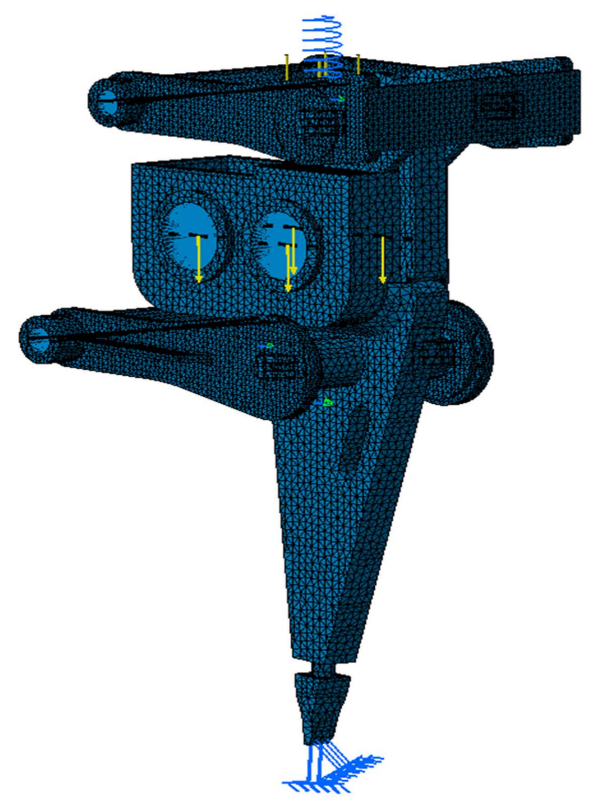

Figure 3. Static FEA model of exciter.

Table 1. Automatic meshing quality report.

\begin{tabular}{cccccc}
\hline Criterion & Good & Poor & Bad & Worst & Average \\
\hline Stretch & $10,305(98.56 \%)$ & $147(1.41 \%)$ & $4(0.04 \%)$ & 0.028 & 0.532 \\
Aspect Ratio & $6726(64.33 \%)$ & $3665(35.05 \%)$ & $65(0.62 \%)$ & 56.71 & 2.430 \\
\hline
\end{tabular}

Table 2. Manual meshing quality report.

\begin{tabular}{cccccc}
\hline Criterion & Good & Poor & Bad & Worst & Average \\
\hline Stretch & $163,965(100.00 \%)$ & $8(0.00 \%)$ & $0(0.00 \%)$ & 0.114 & 0.637 \\
Aspect Ratio & $154,321(94.11 \%)$ & $9645(5.88 \%)$ & $7(0.00 \%)$ & 14.300 & 1.895 \\
\hline
\end{tabular}




\section{Static Calculation Results Analysis}

\subsection{Displacement Result Analysis}

Determine the above conditions, the use of solver CATIA solution to calculate the displacement of the exciter component cloud. Observe and analyze the deformation of the exciter. Figure 4 shows the parallel displacement vector diagram of the exciter. It can be seen from Figure 4 that the maximum displacement of exciter in static analysis is $0.69 \mathrm{~mm}$, and the position occurs behind the right side of gearbox [13]. Among them, Figure 5, Figure 6 and Figure 7 respectively show the displacement distribution of the exciter in the $\mathrm{X}, \mathrm{Y}$ and $\mathrm{Z}$

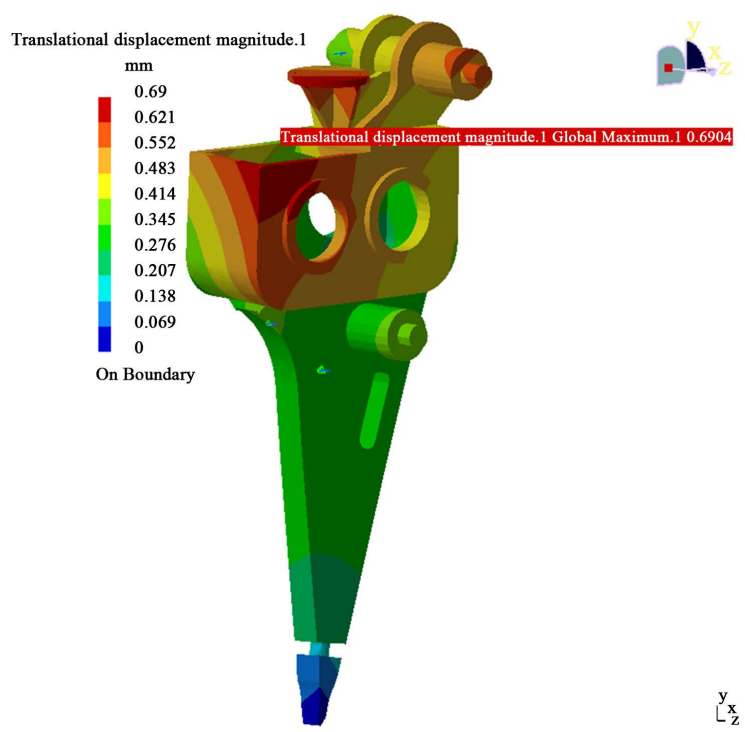

Figure 4. Parallel displacement vector.

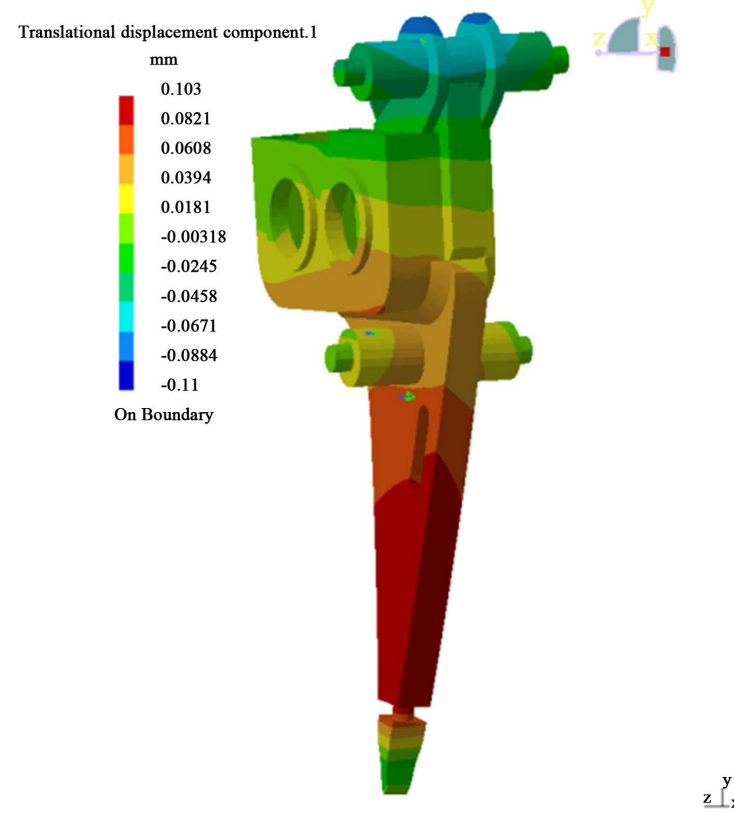

Figure 5. Displacement distribution in X direction. 


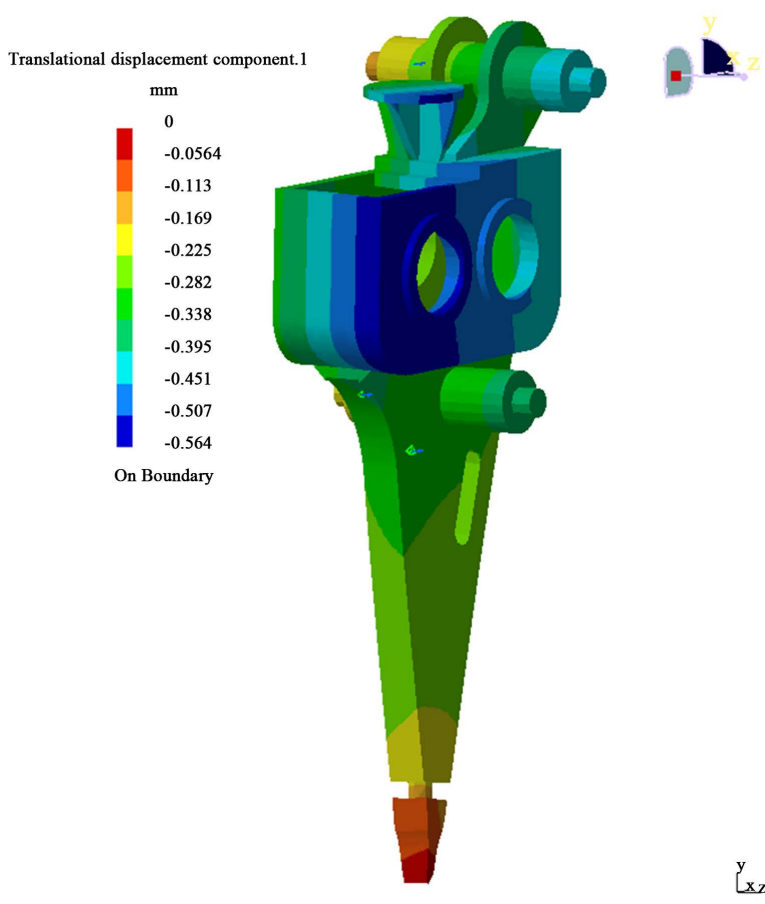

Figure 6. Y-direction displacement distribution.

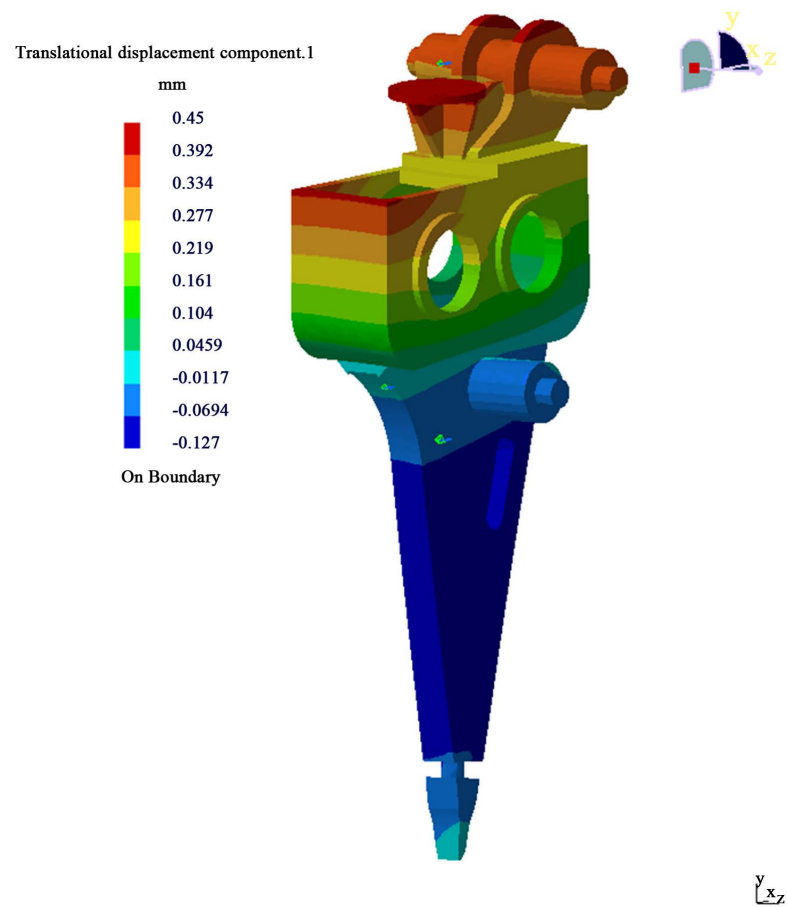

Figure 7. Z-direction displacement distribution.

directions, and the large displacements are $0.11 \mathrm{~mm}, 0.564 \mathrm{~mm}$ and $0.45 \mathrm{~mm}$ respectively. From the $\mathrm{X}, \mathrm{Y}$ and $\mathrm{Z}$ direction of the displacement distribution point of view, the displacement along the $\mathrm{Y}$ the largest change in the amount of displacement in the $\mathrm{Z}$ direction, the smallest amount of change for the $\mathrm{X}$ direction [14]. 
Based on the above analysis results, the following conclusions can be drawn: In the static analysis, the exciter exerts the largest deformation along the $\mathrm{Y}$ direction (vertical ground direction) and the second along the $\mathrm{Z}$ direction (transverse direction) under the action of exciting force and load [15]. The smallest amount of deformation for the $\mathrm{X}$ (vertical). Therefore, the larger vertical and horizontal deformation will cause the exciter to be deformed along the YZ plane. Actual work of the exciter after a long time after the emergence of the knife along the YZ surface bending phenomenon also further verified the displacement analysis [16].

\subsection{Stress Analysis Results}

Through static analysis, see the exciter equivalent stress map. As shown in Figure 8 , the equivalent stress of the exciter shows that the maximum stress occurs at the tip of the tooth, and the stress is as high as $406 \mathrm{Mpa}$. Among them, the local stress is concentrated in the teeth-knife row at the junction, the part of the stress value is mainly $200 \mathrm{Mpa}-400 \mathrm{Mpa}$, solid points in Figure 9 gives the local stress concentration of the specific location [17].

The maximum stress for pull-up and pull-down brackets, respectively, is shown as 52.7 Mpa and 67.5 Mpa. As shown in Figure 10 and Figure 11, where the maximum stress of the pull-up bracket is concentrated in the inner stiffener, the maximum stress of the pull-down bracket is concentrated in the outer stiffener. Because of the symmetrical restraint and the symmetrical design of the upper and lower brackets in the finite element model of the exciter, the analysis of the parallel displacement vector of the exciter is shown in Figure 4: when the exciter is deformed in the $\mathrm{Z}$ direction (That is, transverse deformation), then

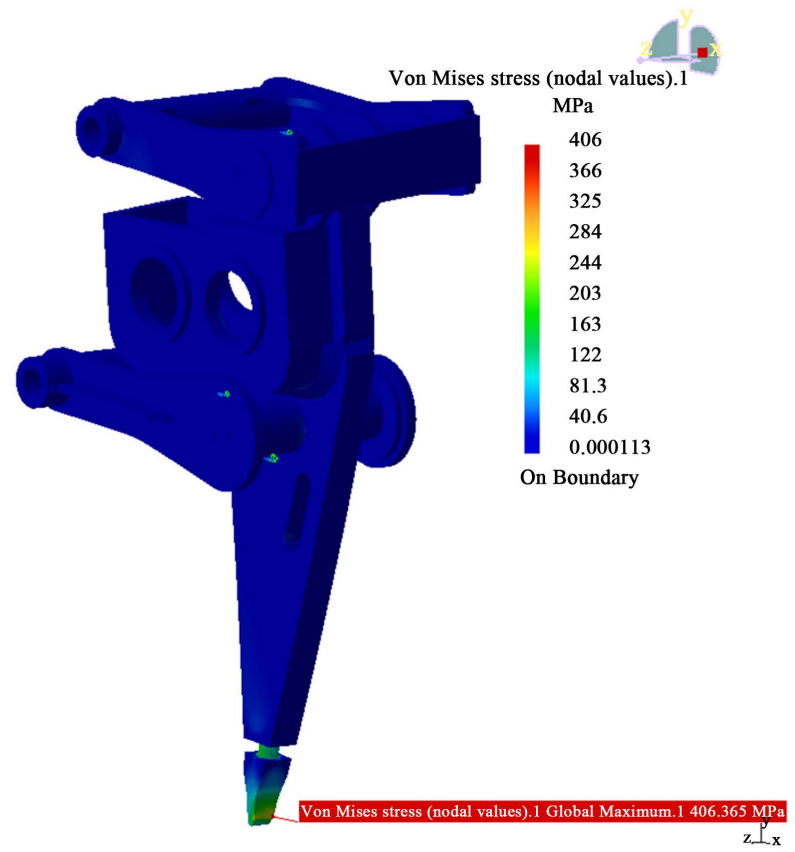

Figure 8. Equivalent stress diagram. 


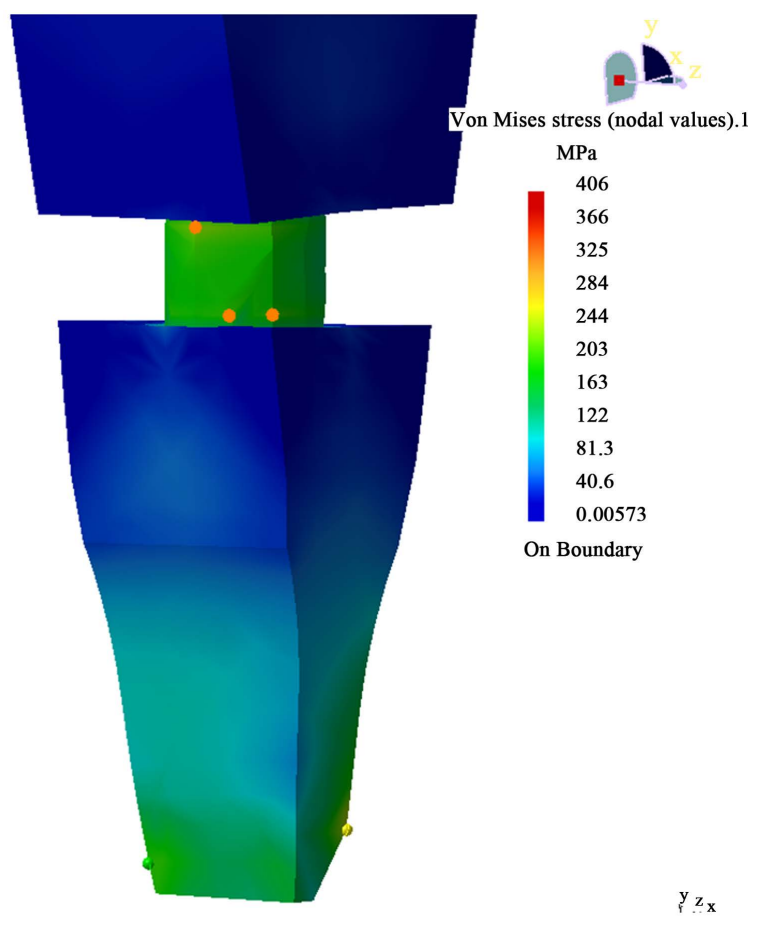

Figure 9. Local stress concentration diagram.

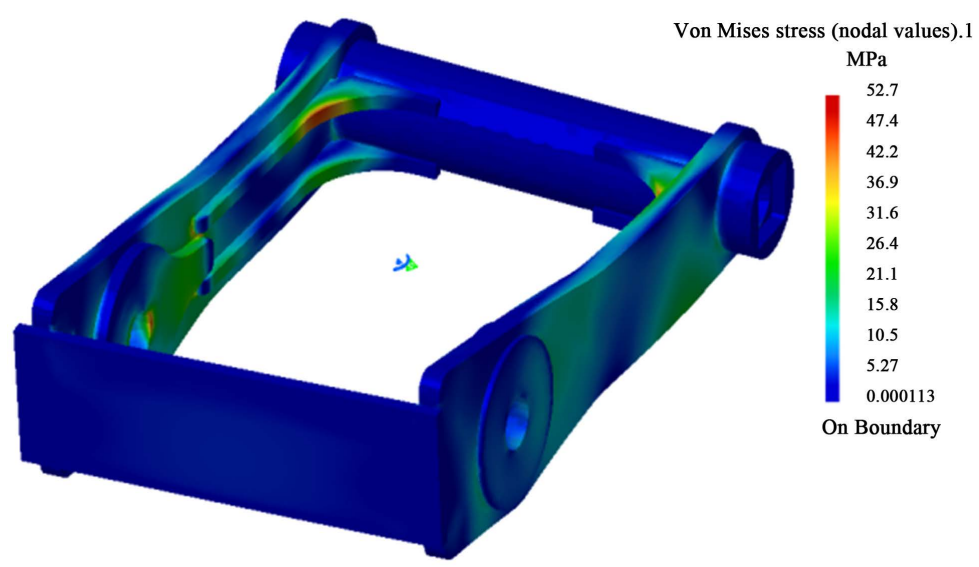

Figure 10. Pull-out bracket equivalent stress diagram.

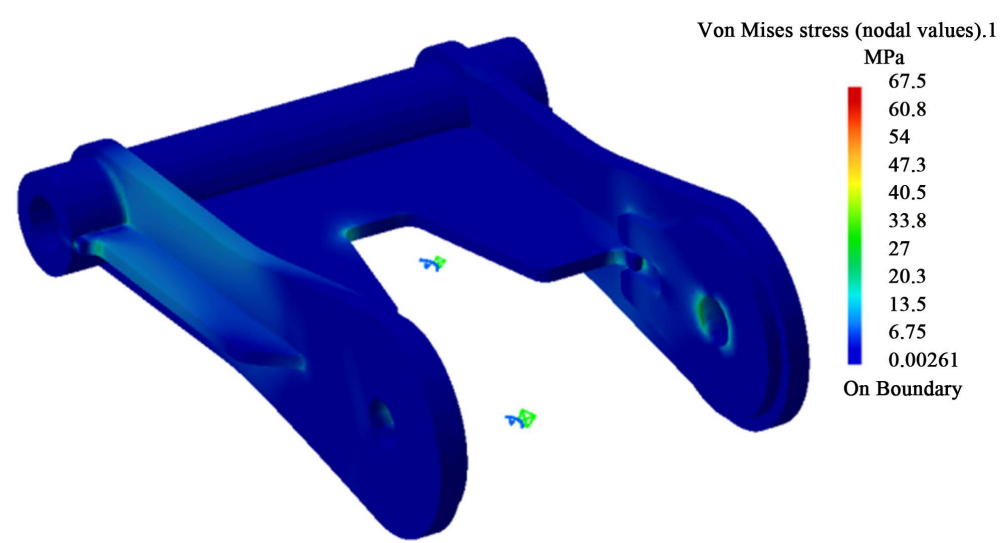

Figure 11. Pull-out bracket equivalent stress diagram. 
the maximum stress of the pull-up bracket and pull-down bracket may completely reach 2 times of the existing analysis value. That is, up to $105.4 \mathrm{Mpa}$ pull the maximum stress stent, pull the stent maximum stress up to $135 \mathrm{Mpa}$ [18].

According to Figures 8-11, it can be seen that the stress concentration area of the high-frequency breaker mainly strengthens in the bucket teeth, Ribs and pull-down braces. The pull-bracket and pull-down bracket stress concentration is relatively small, the body will not be the general body of these parts yield equipment damage [19].

Based on the above allowable stress values and stress analysis results, there is no risk of material failure in the stress concentration zones of the rapiter assembly pull-up bracket and pull-down bracket, and the safety factor is 2.96 to 3.79 , but for the tooth-to-knife row connection At its maximum equivalent stress value of $235.333 \mathrm{Mpa}$ is much greater than the allowable stress value of $188.92 \mathrm{Mpa}$, of course, this maximum equivalent stress value appears in the exciter itself excitation force and $10 \mathrm{t}$ load double load, is an extreme condition. It is also this extreme working condition that exposes the design flaws of the tooth-knife row connection, which makes the part with serious risk of failure. Therefore, it is necessary for the new design of the tooth-knife row connection to be optimized later.

\section{References}

[1] Zhu, J.X. and Zou, X.F. (2001) Domestic and Foreign Research Status of Hydraulic Breakers and Development Trends. Rock Drilling Machinery Pneumatic Tools, No. 4, 33-38.

[2] Shi, X.S. (2012) Research on the Key Technology of Hydraulic Breaker. Zhejiang University, Hangzhou, 1-2.

[3] Tong, X.D. (2014) Overview of the Development of High Frequency Crusher at Home and Abroad. Rock Drilling Machinery Pneumatic Tools, No. 3, 1-7.

[4] Wang, K.L. (2015) Development Status and Research of High Frequency Breaker. Micollurgical Machinery, 43, 1-3.

[5] Hu, K.J. (2014) The Navigator of New Breaker-Vibroripper SV-80. Science Publishing Group Service Invoice, 2, 32-35.

[6] Hu, K.J. (2015) High Frequency Breaker Troubleshooting and Precautions. Machine Tool and Hydraulics, 43, 188-190.

[7] Zhang, D.J. (2008) Current Status and Classification of Domestic Hydraulic Hammer. Jiangsu Metallurgical, No. 3, 4-6.

[8] Yang, X.L. (2000) Meso-Mechanism of Rock Burst Damage Fracture. Explosion and Shock, 20, 247-252.

[9] Lan, Y.F. (2002) The Status Quo and Development of Pile-Driving Machinery in Our Country. Construction Mechanization, 2, 13-16.

[10] Jiang, S. and Yan, X.Z. (2003) Virtual Prototyping Technology and Its Application Prospects in China. Machinery, 30, 4-6.

[11] Gao, J.W. and Gao, J.F. (2013) High Frequency Vibratory Hammering Impacting Machine: China, 103233490A.

[12] Wang, K. and Yang, X.M. (2008) Overview of Virtual Prototype Technology. New 
Technology and New Technology, No. 3, 29-33.

[13] Wang, L. (2010) Research on the Virtual Prototype Technology of Hydraulic Breakers. Shanghai University of Engineering Science, Shanghai, 4-6.

[14] Xi, J.J. (2006) Development and Application of Virtual Prototyping Technology. Journal of Manufacturing Automation, 28, 19-22.

[15] Cao, G.J. and Fan, Y.X. (2011) A High-Frequency Breaker: China, 201110081337.6.

[16] Cao, Y. and Fan, Y.X. (2012) A High-Frequency Breaker: China, 202590877U.

[17] Tang, Z.S. (2011) Hydraulic Resonance Crusher: China, 201020144576.2.

[18] Meng, X.S. (2014) Overview of High-Frequency Hammer. Construction Machinery Digest, No. 4, 79-80.

[19] Quark Studios (2001) CATIA Example. Science Press, Beijing. 